\title{
Team-Based Learning in Chemistry Courses with Laboratory Sessions
}

\author{
Aires-de-Sousa, Joao; Cardoso, M. Margarida; Ferreira, Luisa M.; Lima, Joao C.; \\ Noronha, Joao P.; Nunes, Ana V.M.; Nunes da Ponte, Manuel \\ REQUIMTE, Departamento de Química, Faculdade de Ciências e Tecnologia, \\ Universidade Nova de Lisboa, 2829-516 Caparica, Portugal.
}

\begin{abstract}
The implementation of Team-Based Learning (TBL, http://www.teambasedlearning.org) in one-semester undergraduate courses of chemistry offered to first year students is reported. TBL is an active learning instructional strategy heavily relying on small group interaction. Teaching lab classes in a TBL context presented a specific challenge, as decisions were required about their role in the global framework and the possibility of incorporating lab activities as "teamwork". The design of lab sessions as TBL team application activities is here also illustrated, both for a course of General Chemistry and a course of Organic Chemistry. TBL dramatically improved students class attendance and participation. Its implementation has provided a unique opportunity for the pedagogical development of teaching staff. A moderate number of students reported discomfort with TBL: the requirement of individual preparation before classes and the impact of team participation in the final grade is indeed a new ground for most students, often perceived as a troubling deviation from the common social paradigm of the learning process. The role of the instructor as a facilitator of individual and team work, and the clear explanation of the method are thus of utmost relevance.
\end{abstract}

Keywords: Team-Based Learning; Chemistry; Laboratories; Flipped Classroom; Just-in-Time Teaching; Active Learning. 


\section{Introduction}

Pedagogical innovation is becoming a key aspect in assessing the quality of Universities (Abbott et al., 2014) due to new requirements of labour markets, new sources of information for intellectual production and new possibilities for social participation. At the same time, insights about the neural processes of learning demand new strategies of teaching. Pedagogical innovation cannot simply aim at incremental improvements of traditional procedures with no questioning of the paradigms of teaching and learning. Evidence is now available that active learning can yield better educational results than the traditional method based on lectures (Freeman et al., 2014; Waldrop, 2015). Organizational changes are demanded to improve science education with evidence-based teaching practices (Bradforth et al., 2015).

Team Based Learning (TBL, http://www.teambasedlearning.org) is an active learning instructional strategy heavily relying on small group interaction (Michaelsen et al., 2002; Michaelsen \& Sweet, 2008). Most of class time is used for team activities consisting in the application of course content to solve problems. With TBL the student's role is completely changed: it now involves accountability for pre-class preparation, readiness for effective teamwork and peer evaluation.

A TBL course is organized in cycles (corresponding to content units) each one consisting in the Readiness Assurance Process (RAP) and Application Activities. The RAP follows a sequence of five steps: a) pre-class individual study, b) individual Readiness Assurance Test (iRAT), c) team Readiness Assurance Test (tRAT), d) written appeals and e) minilecture. Application activities follow, which are designed according to the "4S" rules: significant problems, specific choices, same problem for all teams and simultaneous report.

TBL has a history of more than 20 years (Haidet $e t$ al., 2014) and has been used in a wide range of fields, including chemistry (Walters, 2012; Yasuhara et al., 2013) - although not much has been reported concerning the incorporation of lab sessions in the TBL framework. In this article we communicate our experience in the implementation of undergraduate chemistry courses using the TBL methodology.

\section{Implementation details}

TBL has been implemented in one-semester undergraduate courses of chemistry offered to first year students: a General Chemistry course for engineering students (Mechanical, Physics, Industrial, Geological and Environmental Engineering) involving ca. 300 students/course, and an Organic Chemistry course for Applied Chemistry students with ca. 30 students. Students have generally no familiarity with TBL or other active learning 
methodology. The number of faculty members involved in each course has varied between 2 and 4 . None of them had previous experience with TBL.

The General Chemistry course has adopted TBL since 2011 and is here described in more detail. Lab sessions presented a specific challenge and were re-designed as team applications incorporated in the TBL framework. The courses typically encompass 14 weeks and are structured in five content units (cycles of 2-3 weeks). Each week has two class sessions of 2 and 3 hours.

Students are organized by the instructor in permanent teams of 5-7 members. Team formation aims at creating maximally diverse and balanced teams, based on students characteristics assessed via a Google form questionnaire (gender, main degree, previous chemistry courses, previous grades, and likelihood of attending all classes). Our specific implementation of the TBL routines are described below.

\subsection{Pre-class individual preparation}

Reading assignments and learning material are made available or listed through the campus Moodle platform (http://moodle.org/) and include a) book chapters (Chang, 2000; Clayden, 2012), b) videos of lectures (http://ocw.mit.edu), c) solved problems, d) on-line studying guides (http://www.mhhe.com/physsci/chemistry/chang7), e) mini-texts, and f) list of learning objectives.

\section{2. iRAT (individual Readiness Assurance Test)}

iRAT are simple multiple-choice tests focusing on the main concepts and their straightforward application. In some cases they are taken in class (and we use Remark software from Gravic, Inc. for automatic grading) but most often we rely on the Moodle system for remote testing previous to the first class of each cycle. Moodle tests have been configured so that students have a time limit of 20-30 min for the 10 multiple-choice tests, questions are randomly chosen from a data bank for each student and must be submitted sequentially, i.e. the answers to the first two questions must be submitted before the next two are displayed, and so on.

\section{3. tRAT (team Readiness Assurance Test)}

The same tests are taken by the teams immediately after the iRATs, or as the first activity of the cycle when iRATs are taken remotely. We use IF-AT answer sheets (http://www.epsteineducation.com) that are self-scoring and provide immediate feedback on each team answer. Answers are indicated by scratching the box corresponding to the chosen option - if this is the correct answer a mark is found. Appeals are allowed (and encouraged) when teams do not agree with the key in the answer sheet. 


\subsection{Mini-lecture}

A 20-30 min mini-lecture caps the Readiness Assurance Process. It is usually based on the correction of the RAT tests, and specifically addresses questions arisen during the tRAT discussions. Depending on when and how RAT tests were taken, the mini-lecture may be shaped by the results, to focus on the most difficult issues - just-in-time teaching.

\subsection{Application Activities}

Application activities were designed or adapted to address the objectives of our courses under the TBL " $4 \mathrm{~S}$ " guidelines. At least one application per session is graded. In the General Chemistry course, beyond the problems requiring numerical calculations, problems were devised to deepen the understanding of concepts and foster discussions within teams. For example, in addition to $\mathrm{pH}$ calculations in acid-base equilibria, an application consists in the identification of the dominant species in each stage of a titration.

Typical applications in the Organic Chemistry course include mechanism-based explanation of observed phenomena, or finding the best reactants and other experimental conditions for the synthesis of a target molecule.

The simultaneous submission of answers by all teams for immediate inter-teams discussion (an " $S$ " requirement) has been accomplished by different procedures. For example, in adapted versions of gallery walk, all teams simultaneously display their answers on the board or at walls. In another setup, answers are submitted via Moodle using students' smartphones and immediate display of aggregated results with a data show projector.

Laboratory sessions play an essential role in most chemistry courses, for the demonstration of chemical concepts, for the acquisition of lab work skills and scientific methods, or as exercises for the practical application of acquired knowledge. The TBL framework provides an opportunity to re-design lab sessions as active learning activities. We adapted old lab works to TBL applications, using essentially the same material and reagents. For example, a session dedicated to acid-base titrations was reshaped so that teams must find the concentration of an unknown acid solution using a strong base with known concentration. This team activity is graded based on the result - how close the reported concentration is to the real value. Because teams are typically larger than 5 students, the teams are divided in two: each sub-team validates the results of the other, and the whole team has to arrive at a final answer. Two other sessions (devoted to stoichiometry and chemical kinetics) are set up in the same way.

For a simulated lab application, we programmed a virtual lab of chemical kinetics mounted on a web site. It enables students to choose experimental conditions and to perform experiments (measuring concentrations at real time intervals). Students have to plan experiments, collect data, and use them to determine rate laws and activation energies. 
In the Organic Chemistry course, lab works emphasize the development of individual lab skills and were also integrated as TBL activities. Teams are divided in pairs of students, and to each pair is assigned the synthesis of the same product. Teams have to deliver the maximum possible amount of bulk product (resulting from the contributions of all their students) with the highest possible purity. The team is evaluated on the basis of the amount and purity of the product they deliver. In this way, team members are encouraged to cooperate for the common goal of performing the synthesis in the best possible way. During the process, and at the end, the whole team has to decide which contributed products shall be excluded if their purity is not good enough.

\subsection{Peer evaluation}

Peer evaluation within teams reinforces student accountability to each other and is performed at two different moments, one at the middle of the semester (qualitative and with no impact on the final grade), and the other at the end of the semester (consisting in the distribution of points among teammates). The ipeer software (http://ipeer.ctlt.ubc.ca) was installed in a University server and is used for all peer evaluations.

\section{Assessment}

A dramatic improvement in students class attendance and participation has been reported by all instructors. The results obtained in final exams are globally at least as good as with the traditional lecture system. Most instructors report a perception that less students are "left behind" in the first half of the semester. The TBL implementation has provided a unique opportunity for the pedagogical development of teaching staff.

In a 2012 survey among students of our first General Chemistry course implementing TBL, lab sessions (and solved problems) appeared at the top of the "activities/materials most useful for learning". The percentage of students "agreeing" or "strongly agreeing" that TBL was more useful than the traditional system was $42 \%$, and $57 \%$ considered peer evaluations "fair" or "very fair". In a 2017 survey involving 148 students, 77\% of students "agreed" or "strongly agreed" that they were better trained to work in teams than with the traditional method, $46 \%$ that they were better trained to study individually, $24 \%$ that they learned more, $41 \%$ that they were better trained to solve problems, $45 \%$ were more satisfied with the sessions, and $31 \%$ globally preferred TBL to the traditional system. These surveys, as well as informal perceptions, reveal some resistance from a moderate number of students to the new paradigm of learning and teaching.

Our findings are in line with trends observed in the TBL literature (Haidet et al., 2014), namely a consistent greater participation in TBL-based classrooms, better or similar results 
concerning knowledge acquisition (with students at the low end of the class usually benefitting the most), and some reports of lower student enjoyment or satisfaction.

The requirement of individual preparation before classes and the impact of team participation in the final grade is indeed a new ground for most students in our courses, often perceived as a troubling deviation from the common social paradigm of the learning process. The role of the instructor as a facilitator of individual and team work, and the clear explanation of the method are thus of utmost relevance.

\section{Acknowledgments}

The authors thank Faculdade de Ciências e Tecnologia (Universidade Nova de Lisboa) for financial support.

\section{References}

Abbott, A., Zastrow, M., Gibney, E., \& Nordling, L. (2014). The university experiment: Campus as laboratory. Nature, 514(7522), 288-291.

Bradforth, S.E., Miller, E.R., Dichtel, W.R., Leibovich, A.K., Feig, A.L., Martin, J.D., Bjorkman, K.S., Schultz, Z.D., \& Smith, T.L. (2015). University learning: Improve undergraduate science education. Nature, 523(7560), 282-284.

Chang, R. (2000). General chemistry: the essential concepts. Mc Graw Hill.

Clayden, J., Greeves, N., Warren, S., \& Wothers, P. (2012). Organic chemistry. Oxford: Oxford University Press.

Freeman, S., Eddy, S. L., McDonough, M., Smith, M. K., Okoroafor, N., Jordt, H., \& Wenderoth, M. P. (2014). Active learning increases student performance in science, engineering, and mathematics. Proceedings of the National Academy of Sciences, 111(23), 8410-8415.

Haidet, P., Kubitz, K., \& McCormack, W. T. (2014). Analysis of the team-based learning literature: TBL comes of age. Journal on Excellence in College Teaching, 25(3-4), 303333.

Michaelsen, L. K., Knight, A. B., \& Fink, L. D. (Eds.). (2002). Team-Based Learning: A Transformative Use of Small Groups. Greenwood publishing group.

Michaelsen, L. K., \& Sweet, M. (2008). The essential elements of team-based learning. New Directions for Teaching and Learning, 2008(116), 7-27.

Waldrop, M. M. (2015). The science of teaching science. Nature, 523(7560), 272-274.

Walters, D. E. (2012). Team-based learning applied to a medicinal chemistry course. Medical Principles and Practice, 22(1), 2-3.

Yasuhara, T., Konishi, M., Nishida, T., Kushihata, T., Sone, T., Kurio, W., Yamamoto, Y., Nishikawa, T., Yanada, K., \& Nakamura, M. (2013). Practical chemistry education provided by team-based learning (TBL) and peer evaluation. Yakugaku zasshi: Journal of the Pharmaceutical Society of Japan, 134(2), 185-194. 